Erik Talvila*, Department of Mathematics, University of Illinois at

Urbana-Champaign, Urbana, IL 61801. e-mail: talvila@math.uiuc.edu

\title{
LIMITS AND HENSTOCK INTEGRALS OF PRODUCTS
}

\begin{abstract}
When it is known that $\int_{a}^{b} f_{n} \rightarrow \int_{a}^{b} f$ for a sequence of Henstock integrable functions $\left\{f_{n}\right\}$ we give necessary and sufficient conditions for $\int_{a}^{b} f_{n} g_{n} \rightarrow \int_{a}^{b} f g$ for all convergent sequences $\left\{g_{n}\right\}$ of functions of uniform bounded variation. The conditions are easy to apply and involve either the uniform boundedness or uniform convergence of the indefinite integrals of $f_{n}$. The proof uses Stieltjes integrals and applies to bounded or unbounded intervals on the real line. It is shown how to define Stieltjes integrals on unbounded intervals without treating them as improper integrals. The special cases $f_{n} \equiv f$ or $g_{n} \equiv g$ are also examined. The Abel and Dirichlet tests for integrability of a product are obtained as corollaries as well as a form of the Riemann-Lebesgue lemma. And, if $\Phi: \mathbb{N} \rightarrow(0, \infty)$ it is shown what conditions on $\left\{f_{n}\right\}$ and $\left\{g_{n}\right\}$ give $\int_{a}^{b} f_{n} g_{n}=O(\Phi(n))$ as $n \rightarrow \infty$.
\end{abstract}

\section{Introduction}

Let $[a, b]$ be a closed interval in the extended real line $(-\infty \leq a<b \leq+\infty)$. Suppose $\left\{f_{n}\right\}$ is a sequence of functions $f_{n}:[a, b] \rightarrow \mathbb{R}$, each of which has a finite Henstock integral over $[a, b]$, and $\int_{a}^{b} f_{n} \rightarrow \int_{a}^{b} f$ as $n \rightarrow \infty$ for some Henstock integrable function $f:[a, b] \rightarrow \mathbb{R}$. This paper answers the following question. What are necessary and sufficient conditions on $f_{n}$ and $g_{n}:[a, b] \rightarrow \mathbb{R}$ so that $\int_{a}^{b} f_{n} g_{n} \rightarrow \int_{a}^{b} f g$ ? Note that we do not assume $f_{n} \rightarrow f$ but it will generally be necessary to assume $g_{n} \rightarrow g$. It is known that for $\int_{a}^{b} f_{n} g_{n}$ to exist for all integrable $f_{n}$ each $g_{n}$ must be of bounded variation. (The functions of bounded variation are multipliers for Henstock integrable functions.) Clearly some condition involving convergence of $\int f_{n}$ to $\int f$ on subintervals is needed

Key Words: Henstock integral, convergence test, Stieltjes integral

Mathematical Reviews subject classification: 26A39, 26A42

Received by the editors July 24, 1999

* Supported by an NSERC Postdoctoral Fellowship 
since the $g_{n}$ could be characteristic functions of intervals in $[a, b]$. As we will see in Theorem 3.1 below, such additional conditions can take two forms. Let $F_{n}(x)=\int_{a}^{x} f_{n}$ and $F(x)=\int_{a}^{x} f$. If $F_{n} \rightarrow F$ uniformly on $[a, b]$ then $\left\{g_{n}\right\}$

must be of uniform bounded variation in order for $\int_{a}^{b} f_{n} g_{n} \rightarrow \int_{a}^{b} f g$. If $\left\{F_{n}\right\}$ is uniformly bounded then $\left\{g_{n}\right\}$ must be of uniform bounded variation and $V\left(g_{n}-g\right)$ must tend to 0 as $n \rightarrow \infty$. Here, the variation of function $g$ is $V(g)=\sup \sum\left|g\left(a_{i}\right)-g\left(b_{i}\right)\right|$ where the supremum is taken over all finite sets of disjoint intervals $\left(a_{i}, b_{i}\right) \subset[a, b]$. The special cases $f_{n} \equiv f$ or $g_{n} \equiv g$ are dealt with in corollaries to the theorem. The results continue to hold when $f_{n}$ and $g_{n}$ are changed on sets of measure zero and $g_{n} \rightarrow g$ almost everywhere.

If $a=-\infty$ or $b=+\infty$ we treat $[a, b]$ as a compact interval, with topological base the intervals $(\alpha, \beta),[-\infty, \alpha),(\alpha,+\infty]$ for all real numbers $\alpha<\beta$. For $\phi:[-\infty,+\infty] \rightarrow \mathbb{R}$ we demand that $\phi(x) \in \mathbb{R}$ for all $x \in \mathbb{R}, \phi(-\infty) \in \mathbb{R}$ and $\phi(+\infty) \in \mathbb{R}$. For a function to be continuous on $[-\infty,+\infty]$ it must equal its limits at $\pm \infty$. Thus, no definition of the functions $x \mapsto x$ and $x \mapsto \sin x$ can make these functions continuous on $[-\infty,+\infty]$. When there is no confusion write $\infty$ in place of $+\infty$. With this point of view theorems used below such as Bolzano-Weierstrass and integration by parts apply on unbounded intervals. The value of $\phi$ at $\pm \infty$ is immaterial in the Henstock integral $\int_{-\infty}^{\infty} \phi$. But, with the Riemann-Stieltjes integral $\int_{-\infty}^{\infty} d \phi$ the value of $\phi$ at the endpoints is its essence. The proof of our limit theorem will involve Riemann-Stieltjes integrals, for which a new definition is given in Proposition 2.1 below.

\section{Stieltjes Integrals}

The following result shows how to handle Stieltjes integrals on unbounded intervals. For a Henstock integral on $[0, \infty]$, the tag for the last interval $\left[x_{N-1}, \infty\right]$ is taken to be $\infty$ and the corresponding term in the Riemann sum is simply ignored. With a Stieltjes integral this term must be retained. We take the tag for this interval to be $\infty$ and force $x_{N-1}$ to be sufficiently large. Let $\delta:[0, \infty] \rightarrow(0, \infty)$. We will always write tagged partitions of $[0, \infty]$ in the generic form $\mathcal{P}=\left\{\left(z_{i},\left[x_{i-1}, x_{i}\right]\right)\right\}_{i=1}^{N}$, where $z_{i} \in\left[x_{i-1}, x_{i}\right]$, $0=x_{0}<x_{1}<\cdots<x_{N}=\infty$ and $z_{N}=\infty$. Define $\mathcal{P}$ to be $\delta$-fine if $\left(z_{i}-\delta\left(z_{i}\right), z_{i}+\delta\left(z_{i}\right)\right) \supset\left[x_{i-1}, x_{i}\right]$ for $1 \leq i \leq N-1$ and $x_{N-1}>1 / \delta(\infty)$. A regulated function has a left and right limit at each point. Note that a function of bounded variation is regulated. Part iii) below is given in [13], p. 187.

Proposition 2.1. Let $F$ and $g$ be real valued functions on $[0, \infty]$ with one regulated and the other of bounded variation. The following definitions of 
$\int_{0}^{\infty} F d g=A \in \mathbb{R}$ are equivalent.

i) For each $\epsilon>0$ there is a function $\delta:[0, \infty] \rightarrow(0, \infty)$ so that for any $\delta$-fine tagged partition of $[0, \infty]$ we have $\left|\sum_{i=1}^{N} F\left(z_{i}\right)\left[g\left(x_{i}\right)-g\left(x_{i-1}\right)\right]-A\right|<\epsilon$.

ii) For any strictly increasing continuous function $h:[0,1) \rightarrow[0, \infty)$ satisfying $h(0)=0$ and $\lim _{t \rightarrow 1^{-}} h(t)=+\infty$ we have $\int_{0}^{1} F \circ h d(g \circ h)=A$.

iii) $\lim _{t \rightarrow \infty} \int_{0}^{t} F d g+F(\infty)\left[g(\infty)-\lim _{s \rightarrow \infty} g(s)\right]=A$.

Proof. The hypothesis guarantees the existence of $\int_{0}^{t} F d g$ for all $t \in[0, \infty)$.

Suppose iii) holds. Let $\epsilon>0$. By lemma 9.20 in [9], there exists a function $\delta_{1}:[0, \infty] \rightarrow(0, \infty)$ such that if $0<c<\infty$ and $\mathcal{P}$ is a $\delta_{1}$-fine tagged partition of $[0, c]$ then $\left|\sum_{i=1}^{N} F\left(z_{i}\right)\left[g\left(x_{i}\right)-g\left(x_{i-1}\right)\right]-\int_{0}^{c} F d g\right|<\epsilon$. Take $\delta_{\infty}>0$ small enough so that if $1 / \delta_{\infty}<T<\infty$ then $\left|g(T)-\lim _{s \rightarrow \infty} g(s)\right|<\epsilon /(1+|F(\infty)|)$ and $\left|\lim _{t \rightarrow \infty} \int_{0}^{t} F d g-\int_{0}^{T} F d g\right|<\epsilon$. Let $\delta(x)=\delta_{1}(x)$ for $0 \leq x<\infty$ and $\delta(\infty)=\delta_{\infty}$. Let $\mathcal{P}$ be a $\delta$-fine tagged partition of $[0, \infty]$. Then

$$
\begin{aligned}
& \left|\sum_{i=1}^{N} F\left(z_{i}\right)\left[g\left(x_{i}\right)-g\left(x_{i-1}\right)\right]-A\right| \\
& \leq|| \sum_{i=1}^{N-1} F\left(z_{i}\right)\left[g\left(x_{i}\right)-g\left(x_{i-1}\right)\right]-\int_{0}^{x_{N-1}} F d g \mid \\
& \quad+\left|F(\infty)\left[g(\infty)-g\left(x_{N-1}\right)\right]-F(\infty)\left[g(\infty)-\lim _{s \rightarrow \infty} g(s)\right]\right| \\
& \quad+\left|\lim _{t \rightarrow \infty} \int_{0}^{t} F d g-\int_{0}^{x_{N-1}} F d g\right| \leq 3 \epsilon .
\end{aligned}
$$

Hence we have i).

Now suppose i) holds. Let $\epsilon>0$. We can assume $\delta:[0, \infty] \rightarrow(0, \infty)$ such that $1 / \delta(\infty)<T<\infty$ implies $\left|g(T)-\lim _{s \rightarrow \infty} g(s)\right|<\epsilon /(1+|F(\infty)|)$. Let $1 / \delta(\infty)<T<\infty$. There is a $\delta$-fine tagged partition of $[0, \infty]$ with $x_{N-1}=T$. 
And, there is $A \in \mathbb{R}$ such that

$$
\begin{aligned}
\epsilon & >\left|\sum_{i=1}^{N} F\left(z_{i}\right)\left[g\left(x_{i}\right)-g\left(x_{i-1}\right)\right]-A\right| \\
& \geq\left|\sum_{i=1}^{N-1} F\left(z_{i}\right)\left[g\left(x_{i}\right)-g\left(x_{i-1}\right)\right]-B\right|-\epsilon
\end{aligned}
$$

where $B=A-F(\infty)\left[g(\infty)-\lim _{s \rightarrow \infty} g(s)\right]$. It follows that $\left|\int_{0}^{T} F d g-B\right|<3 \epsilon$. This gives iii).

With a change of variables, iii) becomes

$$
\lim _{t \rightarrow 1^{-}} \int_{0}^{t} F \circ h d(g \circ h)+F \circ h(1)\left[g \circ h(1)-\lim _{s \rightarrow 1^{-}} g \circ h(s)\right]=A .
$$

The proof that ii) is equivalent to iii) is now similar to the above.

There are obvious modifications for other unbounded intervals. When $F$ is continuous and $g$ is of bounded variation we have a Riemann-Stieltjes integral and $\delta$ can be taken to be a constant.

\section{Limit Theorem}

We now present the main theorem.

Theorem 3.1. Let $\left\{f_{n}\right\}$ be a sequence of Henstock integrable functions such that $f_{n}:[a, b] \rightarrow \mathbb{R}$ and $\int_{a}^{b} f_{n} \rightarrow \int_{a}^{b} f$ as $n \rightarrow \infty$ for some Henstock integrable function $f:[a, b] \rightarrow \mathbb{R}$. Define $F_{n}(x)=\int_{a}^{x} f_{n}$ and $F(x)=\int_{a}^{x} f$. Let $\left\{g_{n}\right\}$ be a sequence of functions such that $g_{n}:[a, b] \rightarrow \mathbb{R},\left\{g_{n}\right\}$ is of uniform bounded variation and $\left\{g_{n}\right\}$ converges pointwise on $[a, b]$ to the function $g:[a, b] \rightarrow \mathbb{R}$. Then convergence $\int_{a}^{b} f_{n} g_{n} \rightarrow \int_{a}^{b} f g$ for all such $\left\{g_{n}\right\}$ is equivalent to each of the following:

i) $F_{n} \rightarrow F$ uniformly on $[a, b]$,

ii) $F_{n} \rightarrow F$ on $[a, b],\left\{F_{n}\right\}$ is uniformly bounded on $[a, b]$, under the additional assumption $V\left(g_{n}-g\right) \rightarrow 0$,

iii) $\int_{a}^{b} F_{n} d g_{n} \rightarrow \int_{a}^{b} F d g$. 
Proof. i) Suppose $F_{n} \rightarrow F$ uniformly on $[a, b]$. Since $\left\{g_{n}\right\}$ is of uniform bounded variation and $g_{n} \rightarrow g$ it follows there is a constant $M$ so that $|g| \leq$ $M, V(g) \leq M$ and $\left|g_{n}\right| \leq M, V\left(g_{n}\right) \leq M$ for all $n$. Write $f_{n} g_{n}-f g=$ $\left(f_{n}-f\right) g_{n}+f\left(g_{n}-g\right)$. Integrate by parts $([9]$, Theorem 12.21),

$$
\int_{a}^{b}\left(f_{n}-f\right) g_{n}=g_{n}(b) \int_{a}^{b}\left(f_{n}-f\right)-\int_{a}^{b}\left(F_{n}-F\right) d g_{n} .
$$

It follows that

$$
\left|\int_{a}^{b}\left(f_{n}-f\right) g_{n}\right| \leq M\left|\int_{a}^{b}\left(f_{n}-f\right)\right|+\max _{a \leq x \leq b}\left|F_{n}(x)-F(x)\right| M .
$$

Both expressions on the right tend to 0 as $n \rightarrow \infty$.

Also,

$$
\int_{a}^{b} f\left(g_{n}-g\right)=\left[g_{n}(b)-g(b)\right] \int_{a}^{b} f-\int_{a}^{b} F d g_{n}+\int_{a}^{b} F d g .
$$

The first term on the right tends to 0 since $g_{n} \rightarrow g$ pointwise. As $\left\{g_{n}\right\}$ is of uniform bounded variation and $F$ is continuous on $[a, b]$, we have $\int_{a}^{b} F d g_{n} \rightarrow$ $\int_{a}^{b} F d g$. (The proof of the theorem on page 212 of [13] can be extended to unbounded intervals using Proposition 2.1.) This proves sufficiency.

Now we show it is necessary to assume $F_{n} \rightarrow F$ uniformly on $[a, b]$. Suppose $F_{n} \nrightarrow F$ on $[a, b]$ or $F_{n} \rightarrow F$ on $[a, b]$ but not uniformly. Then there is a sequence in $[a, b]$ on which $F_{n}-F \nrightarrow 0$. The sequence has a convergent subsequence $\left\{y_{n}\right\}_{n \in I}$ defined by the unbounded index set $I \subset \mathbb{N}$ (BolzanoWeierstrass). As $n \rightarrow \infty$ in $I$, we have $F_{n}\left(y_{n}\right)-F\left(y_{n}\right) \nrightarrow 0$ but $y_{n} \rightarrow y$. With no loss of generality we may assume $a<y_{n} \leq y \leq b$.

Let $H$ be the Heaviside step function $(H(x)=1$ for $x \geq 0$ and $H(x)=0$ otherwise). Define $g_{n}(x)=H\left(x-y_{n}\right)$ for $n \in I$ and $g_{n}(x)=H(x-y)$ otherwise. Then $g(x)=H(x-y)$ and $V\left(g_{n}\right)=1$. Let $n \in I$. Then

$$
\begin{gathered}
\int_{a}^{b} f_{n} g_{n}=\int_{y_{n}}^{b} f_{n}=F_{n}(b)-F_{n}\left(y_{n}\right) \\
\int_{a}^{b} f g=\int_{y}^{b} f=F(b)-F(y) .
\end{gathered}
$$


Since $F_{n}(b) \rightarrow F(b)$ and $F$ and $F_{n}$ are continuous this gives our contradiction and proves i).

To prove ii), suppose that $F_{n} \rightarrow F$ pointwise, $\left|F_{n}\right| \leq M$ for all $n$ and $V\left(g_{n}-g\right) \rightarrow 0$. Write $f_{n} g_{n}-f g=f_{n}\left(g_{n}-g\right)+\left(f_{n}-f\right) g$. Integrate by parts,

$$
\begin{aligned}
\left|\int_{a}^{b} f_{n}\left(g_{n}-g\right)\right| & =\left|\left[g_{n}(b)-g(b)\right] \int_{a}^{b} f_{n}-\int_{a}^{b} F_{n} d\left(g_{n}-g\right)\right| \\
& \leq\left|g_{n}(b)-g(b)\right| M+M V\left(g_{n}-g\right) \\
& \rightarrow 0 \text { as } n \rightarrow \infty
\end{aligned}
$$

Also,

$$
\int_{a}^{b}\left(f_{n}-f\right) g=g(b) \int_{a}^{b}\left(f_{n}-f\right)-\int_{a}^{b}\left(F_{n}-F\right) d g .
$$

We have $\int_{a}^{b} f_{n} \rightarrow \int_{a}^{b} f$. And, since $g$ is of bounded variation, $\left|F_{n}\right| \leq M$ and $F_{n} \rightarrow F$ pointwise, the dominated convergence theorem for RiemannStieltjes integrals applies so that $\int_{a}^{b}\left(F_{n}-F\right) d g \rightarrow 0$ ([13], p. 205). Hence, $\int_{a}^{b} f_{n} g_{n} \rightarrow \int_{a}^{b} f g$.

If there is $c \in(a, b)$ such that $F_{n}(c) \not \rightarrow F(c)$ then let $g_{n}(x)=g(x)=$ $H(x-c)$. Then $V\left(g_{n}\right)=1$ and $V\left(g_{n}-g\right)=0$. And,

$$
\begin{gathered}
\int_{a}^{b} f_{n} g_{n}=F_{n}(b)-F_{n}(c) \\
\int_{a}^{b} f g=F(b)-F(c) .
\end{gathered}
$$

Since $\int_{a}^{b} f_{n} \rightarrow \int_{a}^{b} f$, it follows that $\int_{a}^{b} f_{n} g_{n} \nrightarrow \int_{a}^{b} f g$.

If $F_{n}$ is not uniformly bounded then there is a sequence on which $\left|F_{n}\right| \rightarrow \infty$. With no loss of generality, there is a subsequence $\left\{y_{n}\right\}_{n \in I}$ defined by the unbounded index set $I \subset \mathbb{N}$ so that for $n \in I$ we have $F_{n}\left(y_{n}\right) \geq 1, F_{n}\left(y_{n}\right) \rightarrow$ $+\infty$ and $y_{n} \rightarrow y$ for some $a<y_{n}<y \leq b$. Let $g_{n}(x)=H\left(x-y_{n}\right) / \sqrt{F_{n}\left(y_{n}\right)}$ for $n \in I$ and $g_{n}(x)=0$ otherwise. Then $V\left(g_{n}\right) \leq 1, g=0$ and $V\left(g_{n}-g\right) \rightarrow 0$. For $n \in I$ we have

$$
\int_{a}^{b} f_{n} g_{n}=\frac{F_{n}(b)-F_{n}\left(y_{n}\right)}{\sqrt{F_{n}\left(y_{n}\right)}} \rightarrow-\infty
$$


whereas $\int_{a}^{b} f g=0$.

The proof of iii) follows immediately from (1) and (3).

Corollary 3.2. Suppose $f:[a, b] \rightarrow \mathbb{R}$ is measurable. Then $\int_{a}^{b} f g_{n} \rightarrow \int_{a}^{b} f g$ for all functions of uniform bounded variation $g_{n}:[a, b] \rightarrow \mathbb{R}$ with $g_{n} \rightarrow g$ if and only if $\int_{a}^{b} f$ exists.

This contains a version of the Riemann-Lebesgue lemma: If $f$ is integrable over $[0,1]$ then

$$
\int_{x=0}^{1} f(x) e^{i 2 n \pi x} d x=o(n) \quad \text { as } n \rightarrow \infty \text {. }
$$

Note that the functions $\sin (2 n \pi x)$ and $\cos (2 n \pi x)$ both have variation $4 n$ over $[0,1]$ so $\exp (i 2 n \pi x) / n$ is of uniform bounded variation. This estimate was proven sharp in [15].

Corollary 3.3. Suppose the functions $f_{n}:[a, b] \rightarrow \mathbb{R}$ are integrable and $\int_{a}^{b} f_{n} \rightarrow \int_{a}^{b} f$ for some integrable function $f$. Then $\int_{a}^{b} f_{n} g \rightarrow \int_{a}^{b} f g$ for all functions $g:[a, b] \rightarrow \mathbb{R}$ of bounded variation if $F_{n} \rightarrow F$ on $[a, b]$ and $\left\{F_{n}\right\}$ is uniformly bounded.

Necessary and sufficient conditions are not known for the above case.

Corollary 3.2 includes the Abel test for integrability of a product: If $\int_{a}^{b} f$ exists and $g$ is of bounded variation then $\int_{a}^{b} f g$ exists. We also have a result than can be useful when $\int_{a}^{b} f_{n}$ does not exist:

Corollary 3.4. If $\left|\int_{a}^{x} f_{n}\right| \leq M$ for all $n \geq 1$ and all $x \in[a, b)$; if each $g_{n}$ is of bounded variation; if $\lim _{x \rightarrow b^{-}} g_{n}(x)=0$, uniformly in $n$; if $g_{n} \rightarrow 0$ on $[a, b]$ and if $V\left(g_{n}\right) \rightarrow 0$ then $\int_{a}^{b} f_{n} g_{n} \rightarrow 0$.

Proof. Let $x \in(a, b)$ and fix $n \geq 1$. Integrate by parts,

$$
\int_{a}^{x} f_{n} g_{n}=g_{n}(x) \int_{a}^{x} f_{n}-\int_{a}^{x} F_{n} d g_{n} .
$$

We have $\left|g_{n}(x) \int_{a}^{x} f_{n}\right| \leq\left|g_{n}(x)\right| M \rightarrow 0$ as $x \rightarrow b^{-}$. And,

$$
\int_{a}^{x} F_{n} d g_{n}=\int_{t=a}^{b} F_{n}(t) H(x-t) d g_{n}(t) .
$$


Defining $F_{n}(b)=0$ gives

$$
\lim _{x \rightarrow b^{-}} F_{n}(t) H(x-t)=\left\{\begin{array}{cl}
F_{n}(t), & a \leq t<b \\
0, & t=b
\end{array}\right.
$$

The dominated convergence theorem now shows $\lim _{x \rightarrow b^{-}} \int_{a}^{x} F_{n} d g_{n}=\int_{a}^{b} F_{n} d g_{n}$ for each $n \geq 1$. So, $\int_{a}^{b} f_{n} g_{n}$ exists for all $n \geq 1$. As above, $\left|\int_{a}^{x} f_{n} g_{n}\right| \leq$ $M\left[\left|g_{n}(x)\right|+V g_{n}\right]$. It now follows that $\lim _{n \rightarrow \infty} \lim _{x \rightarrow b^{-}} \int_{a}^{x} f_{n} g_{n}=0$ since $\left|g_{n}(x)\right|$ is uniformly small as $x \rightarrow b^{-}$and $V g_{n} \rightarrow 0$.

Note that it is not assumed here that $F_{n}$ has a limit or that $\int_{a}^{b} f_{n}$ exists. The premise of the corollary implies that $\left\{g_{n}\right\}$ is of uniform bounded variation. The first part of the proof gives the Dirichlet test for integrability of a product. This asserts the existence of $\int_{a}^{b} f g$ given that $\left|\int_{a}^{x} f\right| \leq M$ and that $g$ is of bounded variation such that $\lim _{x \rightarrow b^{-}} g(x)=0$ or $\lim _{x \rightarrow b^{-}} F(x) g(x)$ exists. See [12] and the forthcoming book [3] for other such tests.

Corollary 3.5. Let the functions $f_{n}:[a, b] \rightarrow \mathbb{R}$ be integrable and suppose we have a growth function $\Phi: \mathbb{N} \rightarrow(0, \infty)$. Then $\int_{a}^{b} f_{n} g_{n}=O(\Phi(n))$ for all uniformly bounded functions $g_{n}:[a, b] \rightarrow \mathbb{R}$ of uniform bounded variation if and only if $F_{n}=O(\Phi(n))$ uniformly on $[a, b]$.

Proof. Suppose $\left|g_{n}\right| \leq M,\left|V g_{n}\right| \leq M$ and $F_{n}=O(\Phi(n))$, uniformly on $[a, b]$. As in (2) we have $\left|\int_{a}^{b} f_{n} g_{n}\right| \leq M\left(\left|F_{n}(b)\right|+\max _{a \leq x \leq b}\left|F_{n}(x)\right|\right)=O(\Phi(n))$. To show necessity, we proceed as in case i) of the theorem. If $F_{n}$ is not $O(\Phi(n))$ uniformly on $[a, b]$ then there is a sequence $y_{n} \in[a, b]$ such that $y_{n} \rightarrow y \in[a, b]$ and $F_{n}\left(y_{n}\right) / \Phi(n) \rightarrow+\infty$ as $n \rightarrow \infty$ in $I \subset \mathbb{N}$. Let $g_{n}(x)=H\left(y_{n}-x\right)$ for $n \in I$ and $g_{n}(x)=H(y-x)$ otherwise. This gives $\int_{a}^{b} f_{n} g_{n}=\int_{a}^{y_{n}} f_{n}=F_{n}\left(y_{n}\right) \neq$ $O(\Phi(n))$ as $n \rightarrow \infty$ in $I$.

Remark 3.6. The Alexiewicz norm ([1]) of an integrable function $f$ is defined by $\|f\|=\sup _{a \leq x \leq b}\left|\int_{a}^{x} f\right|$. Condition i) of the theorem can be written $\left\|f_{n}-f\right\| \rightarrow$ 0 . And, in ii), $F_{n}$ being uniformly bounded on $[a, b]$ is equivalent to the uniform boundedness of $\left\|f_{n}\right\|$.

Remark 3.7. Some care is needed in the case of infinite intervals. The conclusion following (3) is essentially Helley's second theorem ([14], p. 233). The editor's appendix (p. 240) contains the example

$$
g_{n}(x)=\left\{\begin{array}{cl}
0, & x \leq n \\
x-n, & n \leq x \leq n+1 \\
1, & x \geq n+1
\end{array}\right.
$$


It is claimed that $\int_{-\infty}^{+\infty} d g_{n} \not \int_{-\infty}^{+\infty} d g$. However, specifying $g_{n}$ at the endpoints $\pm \infty$ corrects this problem. Let $\alpha$ and $\beta$ be any real numbers. Defining $g_{n}(-\infty)=\alpha$ and $g_{n}(+\infty)=\beta$ gives $g(-\infty)=\alpha, g(+\infty)=\beta$ and $g(x)=0$ for $x \in \mathbb{R}$. And, $V\left(g_{n}\right)=|\alpha|+1+|\beta-1|$. Using Proposition 2.1, $\int_{-\infty}^{+\infty} d g_{n}=\beta-\alpha=\int_{-\infty}^{+\infty} d g$. Thus, $\int_{-\infty}^{+\infty} d g_{n} \rightarrow \int_{-\infty}^{+\infty} d g$.

Remark 3.8. The special cases in Corollaries 3.2 and 3.3 are examined from a different perspective in [6], namely Theorems 48 and 49 in Chapter 1, as proved for the wide Denjoy integral (Denjoy-Khintchine). (But, they also hold for the restricted Denjoy integral, which is equivalent to the Henstock integral.) Theorem 49 assumes $f_{n} \rightarrow f$ and requires $\left\{F_{n}\right\}$ to be $U A C G_{*}$ and continuous, uniformly with respect to $n$ (equicontinuous), in order to provide a sufficient condition to give $\int_{a}^{b} f_{n} \rightarrow \int_{a}^{b} f$. In this paper we need $\left\{F_{n}\right\}$ to be uniformly bounded and assume $\int_{a}^{x} f_{n} \rightarrow \int_{a}^{x} f$ for each $x \in[a, b]$. Necessary and sufficient for $\int_{a}^{b} f_{n} \rightarrow \int_{a}^{b} f$ is that $\left\{f_{n}\right\}$ be $\gamma$-convergent to $f([4])$. (See also [10], Theorems 11.1, 11.2, 13.7 and 13.8.) If $f_{n} \rightarrow f$ then sufficient is that $\left\{f_{n}\right\}$ be uniformly Henstock integrable ([9]) or that $\left\{F_{n}\right\}$ be $U A C G_{*}$ and uniformly continuous with respect to $n$ ([6], Theorem 47). See [5] for an example of a sequence of continuous functions that has a uniform limit but is not $U A C G_{*}$. The definitions are given there as well. For $U A C G^{*}$ in [6] and [11], read $U A C G_{*}$ in [5] and [9]. See also [11], Theorems 12.4 and 12.11, for different versions of our Corollaries 3.2 and 3.3.

Remark 3.9. Note that if a sequence of continuous functions converges to a continuous function the convergence is quasi-uniform but need not be uniform. See [7] or [8]. Similarly when the functions are $A C G$. For example, $\phi_{n}(x)=n x \exp (-n x)$ converges to 0 on $[0,1]$ but not uniformly since $\phi_{n}(1 / n)=\exp (-1)$. Hence, the condition in i) is not superfluous.

\section{Examples}

The first example shows $\left\{f_{n}\right\}$ need not have a limit (not even almost everywhere) and that if $f=0$ then the theorem may still apply when $\left\{g_{n}\right\}$ does not have a limit. The second example deals with integrals of derivatives and the third with a convolution where $n$ has been replaced with a continuous variable. A final example involves the Dirichlet test.

Example 4.1. Let $[a, b]=[0,1]$ and let $f_{n}(x)=a_{n} \cos (2 n \pi x)$ where $\left\{a_{n}\right\}$ is a sequence of real numbers. Then $F_{n}(x)=a_{n} \sin (2 n \pi x) /(2 n \pi)$. For all sequences $\left\{a_{n}\right\}$ we have $\int_{0}^{1} f_{n}=0$. If $a_{n}=o(n)$ then $F_{n} \rightarrow 0$ uniformly on $[0,1]$. We can take $f=0$. It is clear from equations (1) and (3) that 
if $f=0$ then $\left\{g_{n}\right\}$ need not have a limit at any point in $[0,1]$, provided $g_{n}(1)$ is bounded. Part i) of the theorem applies and $\int_{0}^{1} f_{n} g_{n} \rightarrow 0$ for any sequence $\left\{g_{n}\right\}$ of uniform bounded variation with $\left\{g_{n}(1)\right\}$ bounded (which is so if $\left\{g_{n}\left(x_{0}\right)\right\}$ is bounded at any fixed point $x_{0}$ in $\left.[0,1]\right)$.

If $a_{n}=O(n)$ then $\left\{F_{n}\right\}$ is uniformly bounded. Part ii) applies and $\int_{0}^{1} f_{n} g_{n} \rightarrow 0$ for any sequence of functions $\left\{g_{n}\right\}$ of uniform bounded variation with $g_{n} \rightarrow g$ and $V\left(g_{n}-g\right) \rightarrow 0$.

If $a_{n} \neq O(n)$ then $\left\{F_{n}\right\}$ need not be bounded and the theorem need not apply. Indeed, let $a_{n}=n^{3}$ and $g_{n}(x)=\cos (2 n \pi x) / n^{2}$. Then $g_{n} \rightarrow 0$ and $V\left(g_{n}\right)=4 / n$ but $\int_{0}^{1} f_{n} g_{n}=n / 2 \nrightarrow 0$.

We remark in passing that $\{\cos (2 n \pi x)\}$ is not uniformly Henstock integrable (i.e., not equi-integrable). (See [9] for the definition.) If $\left\{z_{i}\right\}_{i=1}^{N}$ are the tags of a $\delta$-fine tagged partition of $[0,1]$ then there are positive integers $n$ and $k_{i}$ so that $\cos \left(2 n \pi z_{i}\right) \geq 1 / 2$ for all $1 \leq i \leq N$. By an extension of Dirichlet's approximation theorem (exercise 1 in Chapter 7 of [2]), this inequality can always be solved for some $n \geq 1$ and $0 \leq k_{i} \leq n$. The number $n$ may have to be taken as large as $6^{N}$. For this value of $n$ the Riemann sum is at least as large as $1 / 2$ so $\{\cos (2 n \pi x)\}$ is not uniformly Henstock integrable. I am indebted to Aimo Hinkkanen for supplying the reference to Dirichlet's approximation theorem.

Example 4.2. Let $\beta>\alpha>0$. Define $f_{n}(x)=\frac{d}{d x}\left[\left(x / n^{\beta}\right) \sin \left(n^{\alpha} / x\right)\right]$ when $x \neq 0$ and $f_{n}(0)=0$. For $a=0$ this gives $F_{n}(x)=\left(x / n^{\beta}\right) \sin \left(n^{\alpha} / x\right)$ when $x \neq 0$ and $F_{n}(0)=0$. We have $\left|F_{n}(x)\right| \leq n^{\alpha-\beta} \rightarrow 0$ uniformly on $[0, \infty]$. Let $f=0$ then for any $\left\{g_{n}\right\}$ of uniform bounded variation with $g_{n} \rightarrow g$ we have $\int_{0}^{b} f_{n} g_{n} \rightarrow 0$ for any fixed $0<b \leq \infty$. If $\beta=\alpha$ then part ii) applies on $[0, \infty]$ and part i) applies on bounded intervals but not on $[0, \infty]$ since $F_{n}(x) \rightarrow H(x-\infty)$ and we would then be forced to take $f(x)=\delta(x-\infty)$, the Dirac distribution at $+\infty$.

Example 4.3. Consider the convolution $\gamma(s)=\int_{-\infty}^{\infty} \phi(s-t) \psi(t) d t$ where $s \in \mathbb{R}, \int_{-\infty}^{\infty} \phi$ exists and $\psi$ is real valued on $\mathbb{R}$ and of bounded variation. First suppose $\lim _{t \rightarrow \infty} \psi(t)=\psi_{\infty} \in \mathbb{R}$. Let $g_{s}(t)=\psi(s-t)$. We have $g(t)=$ $\lim _{s \rightarrow \infty} g_{s}(t)=\psi_{\infty}$ for $t \in \mathbb{R}$. Since $V\left(g_{s}\right)=V(\psi)$, Corollary 3.2 gives $\lim _{s \rightarrow \infty} \gamma(s)=\psi_{\infty} \int_{-\infty}^{\infty} \phi$.

Now suppose $\int_{-\infty}^{\infty} \phi=0$. Let $f_{s}(t)=\phi(s-t)$. Then $\int_{-\infty}^{\infty} f_{s}=0$. Take $f=$ 0 . Then $F_{s}(x)=\int_{-\infty}^{x} f_{s}(t) d t=\int_{s-x}^{\infty} \phi \rightarrow 0$ as $s \rightarrow \infty$. Hence, $F_{s} \rightarrow 0$, but perhaps not uniformly. However, $F_{s}$ is uniformly bounded by $\sup _{x \in \mathbb{R}}\left|\int_{x}^{\infty} \phi\right|$. Corollary 3.3 then says $\lim _{s \rightarrow \infty} \gamma(s)=0$. This agrees with the previous part of this example when $\int_{-\infty}^{\infty} \phi=0$. 
Example 4.4. Let $\phi:[0,1] \rightarrow \mathbb{R}$ be integrable. Define $f(x)$ to be $\phi(x \bmod 1)$ if $2 n \leq x<2 n+1$ for some $n \in \mathbb{N}_{0}$ and $f(x)=-\phi(x \bmod 1)$ otherwise. If $g$ is of bounded variation and $g \rightarrow 0$ at infinity then $\int_{0}^{\infty} f g$ exists. Note that $\int_{0}^{\infty} f$ exists only if $\phi=0$ almost everywhere. Special cases are $\int_{1}^{\infty} \sin (x) x^{-p} d x$ and $\int_{1}^{\infty} x^{q} \sin \left(x^{p}\right) d x$ for $p>0$ and $q<p-1$.

\section{References}

[1] A. Alexiewicz, Linear functionals on Denjoy-integrable functions, Colloq. Math. 1(1948), 289-293.

[2] T. M. Apostol, Modular functions and Dirichlet series in number theory, New York, Springer-Verlag, 1990.

[3] R. G. Bartle, A Modern Theory of Integration, Providence, American Mathematical Society.

[4] R. G. Bartle, A convergence theorem for generalized Riemann integrals, Real Anal. Exchange 20(1994/95), 119-124.

[5] B. Bongiorno, L. Di Piazza and V. Skvortsov, Uniform generalized absolute continuity and some related problems in H-integrability, Real Anal. Exchange 19(1993/94), 290-301.

[6] V. G. Čelidze and A.G. Džvaršeǐšvili, The theory of the Denjoy integral and some applications (trans. P.S. Bullen), Singapore, World Scientific, 1989.

[7] N. Dunford and J.T. Schwartz, Linear operators, vol. I, New York, Interscience, 1958.

[8] R. A. Gordon, When is a limit function continuous?, Math. Mag. 71(1998), 306-308.

[9] R. A. Gordon, The integrals of Lebesgue, Denjoy, Perron, and Henstock, Providence, American Mathematical Society, 1994.

[10] R. Henstock, Lectures on the theory of integration, World Scientific, Singapore, 1988.

[11] P-Y. Lee, Lanzhou lectures on Henstock integration, Singapore, World Scientific, 1989. 
[12] J. Mawhin, Analyse-fondements, techniques, évolution, De Boeck Université, Brussels, 1992.

[13] R. M. McLeod, The generalized Riemann integral, Washington, The Mathematical Association of America, 1980.

[14] I. P. Natanson, Theory of functions of a real variable, vol. I (trans. L.F. Boron, ed. E. Hewitt), New York, Ungar, 1955.

[15] E. C. Titchmarsh, The order of magnitude of the coefficients in a generalized Fourier series, Proc. London Math. Soc., Series 2 22(1923/1924) xxv-xxvi (November 9, 1922). 